\title{
SUR L'UTILISATION DU VERT DE MALACHITE EN THERAPEUTIQUE PISCICOLE
}

\author{
établie par M. J.-P. GERARD, Docteur Vétérinaire \\ Laboratoire d'Ichtyopathologie de I'INRA \\ 78850 THIVERVAL-GRIGNON
}

\section{Présentation :}

Le vert de malachite est un colorant se présentant sous forme de cristaux verts, aux reflets métalliques, très solubles dans l'eau. Bien que la solution ait une couleur proche de celle du sulfate de cuivre, ce corps chimique est exempt de cuivre.

Seul, le vert de malachite pur sera employé en thérapeutique pisciaire, la présentation destinée à la teinturerie est à rejeter, car elle contient du chlorure de zinc, toxique pour le poisson.

Indications :

Le vert de malachite est surtout actif contre les champignons. Son emploi préventif évitera le développement de ces parasites. Comme pour toutes les autres médications curativement utilisées, les résultats obtenus seront moins intéressants.

\section{Toxicité :}

Aux doses thérapeutiques indiquées, le vert de malachite n'est pas toxique pour les poissons à condition de respecter la durée, la dose pour une température déterminée de l'eau.

\section{Posologie :}

1 - Pour les bains concentrés (œufs embryonnés).

5 à 10 ppm (1), soit 5 à 10 grammes par mètre cube d'eau, ou 5 à $10 \mathrm{mg}$ par litre. Bains de 20 à 30 minutes dans de l'eau dont la température est inférieure à $15^{\circ}$.

2 - Pour les bains dilués (traitement des alevins, truitelles et truites).

$1 \mathrm{ppm}$, soit 1 gramme par mètre cube ou 1 milligramme par litre. Bains de 40 à 60 minutes.

Mode d'emploi :

Il est nécessaire de préparer une solution-mère qui sera ensuite répartie dans le bassin à traiter.

(1) ppm : partie par million, soit un gramme par mètre cube ou un milligramme par litre. 
Cette solution peut être préparée avec de l'eau, mais pour obtenir une meilleure dilution, elle peut être réalisée avec de l'alcool à $70^{\circ}$. Nous conseillons une solution au $1 / 100$ e dans l'alcool à $70^{\circ}$, c'est-à-dire qu'il faut mélanger 10 grammes de vert de malachite pur dans un litre d'alcool à $70 \circ$.

\section{A - Bains concentrés :}

Ces bains sont réservés essentiellement au traitement des œufs embryonnés. Ils seront effectués durant 20 minutes à la dose de 5 à $10 \mathrm{ppm}$. En utilisant la solution-mère au $1 / 100$ e dans l'alcool à $70 \circ$, il faut 1 cuillerée à café de cette solution (soit $5 \mathrm{ml}$ ) pour 10 litres d'eau pour obtenir un bain à $5 \mathrm{ppm}$.

\section{B - Bains dilués :}

10) Traitement dans les auges d'alevinage.

Le volume d'eau dans l'auge est d'abord déterminé.

Une solution concentrée qui permettra d'obtenir dans l'auge la concentration finale de 1 pour 1000000 est préparée dans un arrosoir.

En employant la solution-mère au $1 / 100$ e dans l'alcool à $70 \circ$, il faut mettre 1 cuillerée à café de cette solution (soit $5 \mathrm{ml}$ ) par 50 litres d'eau.

L'arrivée d'eau étant fermée, la solution concentrée est répartie dans l'auge puis brassée à l'aide d'un balai propre, pour obtenir une solution homogène. Après 20 minutes, les $2 / 3$ de l'eau sont vidangés et l'arrivée de l'eau rétablie.

2) Traitement dans les bassins d'élevage.

Nous conseillons de procéder de la façon suivante :

10) Evaluer le volume en mètres cubes d'eau à traiter.

20) Peser le poids de vert de malachite nécessaire, soit autant de grammes que de mètres cubes.

30) Préparer une solution-mère dans un récipient, un fût de 50 à 100 litres muni d'un robinet convient parfaitement.

40) Faire couler dans l'entrée d'eau du bassin la solution-mère en brassant énergiquement.

50) Dès que la vague de colorant atteint la sortie du bassin, couper l'arrivée d'eau.

60) Laisser 15 à 20 minutes au moins les poissons dans ce bain. en les surveillant afin de rétablir le courant d'eau si des symptômes d'asphyxie se manifestaient.

\section{Périodicité :}

Les traitements au vert de malachite peuvent être effectués tous les 8 à 10 jours, en alternance avec les bains de sulfate de cuivre (voir fiche technique no 0.2). Après la ponte, les géniteurs pourront être traités tous les 3 jours pendant une période de 12 à 15 jours.

$\mathrm{Si}$ les bains préventifs ne sont pas pratiqués dans la pisciculture, le vert de malachite doit être employé aussitôt que quelques poissons présentent des lésions de mycose. Dans ce cas, ils doivent être poursuivis tous les 2 ou 3 jours jusqu'à disparition complète des lésions. Il est exclu d'attendre un résultat favorable d'un seul traitement effectué sans conviction ni esprit de suite. 